\title{
Los desafíos de la nanotecnología para el "desarrollo" en Argentina
}

\author{
Maximiliano Facundo Vila Seoane*
}

RESUMEN: En el artículo se delinean las principales características de la política pública para la nanotecnología en Argentina y el tipo de modelo de desarrollo al que contribuye. Se describen los factores que intervinieron en la promoción de esta política, como la experiencia pionera de EUA, organismos internacionales de crédito y la comunidad científica. A su vez, se bosqueja el modelo analítico de intervención gubernamental, el Sistema Nacional de Innovación, junto con sus debilidades. Más allá de esto, en el artículo se detallan los principales hitos en la conformación de la política de nanotecnología y algunas tensiones en su implementación. Entre ellas: la disputa por su definición; las interacciones entre grupos de investigación y desarrollo y empresas; y los principales desafíos detectados en su implementación. Se concluye con sugerencias para incluir actores de la sociedad civil en el diseño de políticas de nanotecnología, y de ciencia y tecnología en general, para que puedan contribuir a resolver problemas sociales y ambientales del país.

PALABRAS CLAVE: nanotecnología, Argentina, desarrollo, sistema nacional de innovación.

ABSTRACT: This article describes the main features of a public policy for nanotechnology in Argentina and the type of development model that it contributes to. It explains the main factors that inspired the policy, such as the pioneering experience of the US, international financial organizations and the scientific community. In turn, it outlines the analytical model of government intervention, the National System of Innovation, considering its weaknesses. Beyond this, the article details the major milestones that shaped the policy and some tensions in its implementation. Among them: The dispute over its definition; the challenges in the interactions between research and development groups and firms; and key hurdles identified in its implementation. It concludes with suggestions to include civil society actors in policy design processes in nanotechnology, and science and technology in general, so they can enrich approaches to solve social and environmental problems of the country.

KEYWORDS: nanotechnology, Argentina, development, national innovation system.

\section{Introducción}

A partir del 2001, la nanotecnología fue incorporada en la agenda política de diversos países, siguiendo la creación de la iniciativa nacional de Estados Unidos para promover el área. Los motivos detrás de esta decisión se encuentran en las potencialidades económicas asignadas a la nanotecnología. Entre ellas, el ser una tecnología de propósito general, es decir, aplicable en diversos sectores económicos, inclusive capaz, para los más audaces, de propiciar una nueva revolución industrial (MINCyT, 2009: 2). Al tener dicho

* Center for Development Research (ZEF), Bonn, Alemania. Center for Development Research. ZEFA Department. Walter-Flex-Str. 3. D-53113 Bonn, Alemania. Correspondencia: (maxvila@uni-bonn.de). 
potencial transformador, tal como en su momento se anunció para las TICs o las biotecnologías, se comprende el interés de distintas naciones por generar capacidades en el área, a fin de obtener beneficios económicos.

El avance de los conocimientos en nanotecnología sin duda se apoya en décadas de trabajo científico y tecnológico realizado en diversas partes del mundo. Pero este esfuerzo previo no es suficiente para explicar la introducción del tema en la política pública, ni para comprender de qué forma la misma se ha manifestado. Entender estos procesos requiere un análisis de las relaciones entre las políticas de ciencia, tecnología e innovación (CTI) con modelos de "desarrollo". ${ }^{1}$ En este sentido, en los últimos 10 años, varios países de la región han adoptado como marco analítico para sus políticas al Sistema Nacional de Innovación (SNI). Por ejemplo, Argentina, Brasil, Perú, entre otros. Se trata de un marco de referencia que directa o indirectamente guía la forma de organización de los recursos, al suponer que la riqueza de las naciones proviene de la innovación en empresas. Aunque este concepto, surgido en un contexto europeo y estadounidense, presenta dificultades para lidiar con otras realidades donde los perfiles socioproductivos son diferentes.

Argentina es un ejemplo de estas tendencias. Tanto la nanotecnología, como el SNI se utilizan en las políticas de CTI en la última década. En tal contexto, en este trabajo se responde a las siguientes dos preguntas: ¿cuáles han sido los principales eventos, tensiones y desafíos del estímulo de la nanotecnología en Argentina?, y, ¿qué tipo de proyecto político se instaló en el país para la promoción de la nanotecnología? Para responderlas, el trabajo sintetiza y en algunos casos actualiza los resultados de dos investigaciones previas sobre la temática en Argentina ${ }^{2}$ (Vila Seoane y Rodríguez, 2012; Vila Seoane, 2011). En cuanto a la organización del trabajo, en la segunda sección se describen los tres factores que impulsaron una política de nanotecnología en Argentina y los principales hitos durante la primera década de apoyo. En la tercera sección se delinea el tipo de modelo de "desarrollo" con el que comulgan las políticas implementadas. En la cuarta sección se detallan tensiones y desafíos que surgieron a lo largo del proceso. Por último, se sintetizan algunas de las lecciones aprendidas, que podrían ser de utilidad para otros países de América Latina.

\section{Factores detrás de la introducción de la nanotecnología en la política pública de Argentina, y principales hitos}

El origen de la política de nanotecnología en Argentina estuvo influenciada

1 Utilizo las comillas justamente para resaltar que el término "desarrollo" es un término ampliamente debatido y resistido en Latinoamérica y el mundo, debido a su carácter neocolonial y normativo en pos de seguir modelos de países como EUA o algunos europeos.

2 Estos estudios utilizaron tanto técnicas cualitativas (entrevistas con expertos en la temática), como cuantitativas (encuestas con grupos de I+D y empresas de nanotecnología en Argentina), junto con el análisis de información secundaria relevante al tema. 
al menos por los siguientes tres factores. Por un lado, el ímpetu que tuvo la temática a nivel internacional impulsada por Estados Unidos tras crear su iniciativa nacional en 2001. ${ }^{3}$ Otros países siguieron el ejemplo, como China, India o Israel, y también la Unión Europea que creó una estrategia para la promoción de la nanotecnología en 2004 (Vila Seoane, 2011: 48). Esta última medida apuntó a reforzar el liderazgo que países como Alemania, Francia y Reino Unido tienen en la temática.

Un segundo factor fue la influencia de organismos internacionales de crédito. Aquí cabe destacar que si bien en los últimos años hubo un refortalecimiento de la política de CTI en Argentina, varios proyectos de investigación y desarrollo y de modernización tecnológica que implementa la Agencia Nacional de Promoción Científica y Tecnológica ${ }^{4}$ (AGENCIA) se financian a través de créditos otorgados por el Banco Mundial o por el BID. Esto impacta en qué tipo de instrumentos pueden ser diseñados o qué áreas serán financiadas. En off varios entrevistados señalaron esta decisión como una forma pragmática de no depender de la veleidad política para financiar el área. Mientras que para otros es una clara evidencia de falta de soberanía y de una verdadera política de Estado de CTI. No poder definir las prioridades por cuenta propia sin el aval de tales organismos es una sutil relación de dependencia. Al mismo tiempo, muchos de estos organismos internacionales reciben consejos o trabajan en conjunto con consultoras que se dedican a esparcir globalmente estas soluciones, tal como consultoras internacionales como McKinsey, que suelen aconsejar las mismas soluciones a los gobiernos, como si fueran balas de plata. No por nada, en varios países del mundo, las biotecnologías, nanotecnologías y las TICs fueron "detectadas" como prioridades nacionales. Indonesia y Malasia ilustran muy bien este proceso. Si bien ambos países se encuentran muy lejos de América Latina, da la casualidad de que también han identificado junto con McKinsey a las tres mismas áreas como estratégicas para su "desarrollo" (Hornidge, 2012). Los gobiernos suelen aceptar dicha "sugerencia", dado que al tratarse de conceptos con fronteras difusas, se puede tener la esperanza de encontrar una forma de adaptar su interpretación a las realidades locales.

La tercera fue la demanda de las comunidades de investigación nacionales. Ya desde el 2001 demandaron un apoyo activo del Estado para financiar la nanotecnología en el país (Vila Seoane, 2011: 65). A su vez, esta demanda estuvo basada, por un lado en los requisitos que las distintas revistas internacionales (mayoritariamente estadounidense o europeas) que marcan las agendas de investigación sobre la temática, y por el otro, por países que ya habían iniciado la carrera. Especialmente el vecino Brasil, y la relación de amistad-rivalidad que se mantiene aún en diversos campos y que impulsa a Argentina a "no quedarse atrás". Este tercer factor explica por qué la política

$3<$ http://www.nano.gov/>.

4 <http://www.agencia.mincyt.gob.ar/>. 
de nanotecnología ha seguido una orientación inicial de science push, o empujada por la ciencia, si uno utiliza las categorías del denominado, y tantas veces atacado, modelo lineal de innovación. En dicho modelo, se presupone que los resultados de las investigaciones científicas pasan a aplicaciones tecnológicas, y de allí directo al mercado de una forma lineal (Godin, 2006). Y ésta parece ser la orientación de la política, pues se abocó en los primeros años solamente a la financiación de grupos de investigación.

En cuanto a los principales hitos de las políticas de promoción de la nanotecnología en Argentina, a principios del siglo XXI, la política era justamente nano, invisible a la vista de la comunidad nacional. Por reclamo de investigadores, el asunto entró en la agenda pública, aunque lentamente. Cabe resaltar que, a fines del 2001, el país sufrió una de las peores crisis políticas, sociales y económicas de los últimos años. Consecuentemente, las políticas a nivel nacional estaban orientadas a problemas urgentes del corto plazo y no al desarrollo de capacidades estratégicas en áreas de I+D. Si bien se puede disentir de tal decisión, la coyuntura explica en parte el apoyo tardío a las nanotecnologías con respecto a otros países. Recién en 2004, se puede hablar del surgimiento de una política estatal en el área (Andrini y Figueroa, 2008). Primero basada en el apoyo a grupos de I+D a partir de la creación de las primeras redes de investigación en nanotecnología. En el 2005, se incrementó el apoyo con la creación de la Fundación Argentina de Nanotecnología ${ }^{5}$ (FAN). Un proyecto controversial en su diseño original, pues daba la impresión de que las capacidades nacionales de producción de conocimiento se iban a utilizar para producir nano y microestructuras en un laboratorio en suelo estadounidense, bajo el apoyo de Bell labs (Ibid., 2008). Esto, sumado a inconvenientes en el área institucional, generó una fuerte tensión en la pequeña comunidad argentina emergente de nanotecnología (Vila Seoane, 2011). Finalmente, este proyecto original de la FAN fue reducido y destinado sólo a financiar proyectos de emprendedores nacionales. No obstante, el impacto generado fue escaso, dado que pocos de los proyectos que se recibieron eran efectivamente de nanotecnología, o no todos tenían potencial de ser productivos con el capital ofrecido por la fundación.

Desde entonces, el principal foco de acción de la FAN es la difusión de la nanotecnología. En efecto, bianualmente organiza los encuentros Nanomercosur, donde se reúnen mayoritariamente investigadores y empresarios de Argentina, y en menor proporción de países del Mercosur y otros. En total se realizaron 4 eventos, cuya cobertura en diferentes medios ayudó a difundir la temática en el país y a incrementar las redes entre los actores locales e internacionales. Con este trabajo, la FAN ha empezado incipientemente a lograr concientizar sobre las potencialidades de estas nuevas tecnologías a nivel nacional.

$5<$ http://www.fan.org.ar/>. 
A partir del 2007, se puede empezar a hablar de un nuevo periodo en las políticas de fomento. Su inicio coincide con la creación del Ministerio de Ciencia, Tecnología e Innovación Productiva ${ }^{6}$ de Argentina. Y con el uso a nivel de la política pública del marco analítico del Sistema Nacional de Innovación (SNI), originalmente elaborado por autores de Dinamarca, Inglaterra y Estados Unidos (Freeman, 1995; Lundvall et al., 2002; Nelson, 1993). En síntesis, bajo este marco se sostiene que las sociedades en crecimiento son aquellas que apuestan por la innovación, entendida en sus orígenes como la introducción de nuevos productos o procesos al mercado. En su conceptualización, se asume que las empresas son el actor central en los sistemas de innovación. Y que la innovación es un proceso social, donde diferentes instituciones intervienen, entre ellas, pero no las únicas, las del sistema científico y tecnológico. Esta conceptualización coincide con una tesis aceptada en la literatura económica, de que la acumulación de conocimiento y la generación de innovaciones contribuyen al crecimiento económico. No obstante, que esto sólo se derive del buen "funcionamiento" de un "sistema de innovación" ignora o simplifica en exceso trayectorias políticas, culturales y sociales que incentivan procesos de innovación. Por estos motivos, se critica que la innovación se haya convertido en un nuevo mantra para justamente remplazar las críticas que la visión del "desarrollo" suscitó en el mundo entero (Krause, 2013), y específicamente en Latinoamérica (Escobar, 1995).

En el caso argentino, una de las implicaciones de utilizar el marco del SNI fue la reorientación de las políticas de CTI hacia el ámbito empresarial. Ya en 2006, se realizó la primera experiencia de un instrumento que, a pesar de seguir concentrado en grupos de I+D, también incluía empresas en las asociaciones (Vila Seoane, 2011: 68). Pero el cénit se alcanzó en 2010 con el lanzamiento del programa FONARSEC, compuesto por fondos sectoriales millonarios que apuntaron específicamente a crear asociaciones público-privadas entre grupos de investigación y empresas, con el fin de desarrollar productos innovadores en diferentes áreas estratégicas. Una de ellas, la nanotecnología. Cabe resaltar que esta idea se inspiró en los fondos sectoriales brasileños, pero que, a diferencia de ellos, en vez de ser financiados por impuestos a las principales empresas operando en el país de diversos sectores, se financió con créditos del Banco Mundial. Con estas medidas de política, se evidencia la orientación del modelo de "desarrollo".

¿Qué efectos han sido detectados hasta el momento? Una encuesta realizada a grupos de investigación y desarrollo (I+D) y a empresas de nanotecnología en Argentina evidencian un entramado en construcción, y muy superior en tamaño con respecto al prexistente 10 años atrás. Por ejemplo, se obtuvieron respuestas de 81 grupos de I+D que afirmaron estar trabajando en alguna área relacionada con las nanotecnologías, y, a su vez, 23 empresas

$6<$ http://www.mincyt.gob.ar/>. 
indicaron realizar desarrollos nanotecnológicos (Vila Seoane y Rodríguez, 2012). Esta encuesta detectó al menos 629 personas trabajando en grupos de I+D, y al menos unas 103 en empresas. Si bien es una comunidad relativamente chica, si se considera la atracción que la nanotecnología ha generado a nivel mundial, es posible que no hubiese llegado ni siquiera a ese tamaño sin la intervención estatal. La orientación de las políticas gubernamentales en favor del área ha favorecido su incremento, aunque tal vez no tanto ni tan rápido como los mismos miembros de la emergente comunidad en el 2005 esperaban. Otro de los efectos de este último instrumento ha sido el acercamiento de un incipiente número de empresas a grupos de $\mathrm{I}+\mathrm{D}$ en el área de la nanotecnología. Alianzas que 10 años atrás no hubieran sido algo tan común en una nueva área de alto desarrollo científico y tecnológico. No obstante, el impacto en términos de desarrollo económico, en función de los montos invertidos, es algo que aún no ha sido estudiado.

\section{El tipo de modelo de desarrollo de la nanotecnología en Argentina}

Los últimos 10 años de políticas de nanotecnología en el país han conformado lo que denominé como Sistema Nacional de Nanotecnología (Vila Seoane, 2011). En esencia, el nombre alude a un recorte, o subsistema, del concepto analítico del SNI. Aunque en su forma está inspirado en el triángulo de Sábato-Botana para comprender las relaciones de los principales actores que se encuentran trabajando de una u otra forma con nanotecnologías (Sábato y Botana, 1970). Si bien, el discurso durante los últimos años ha estado orientado a fortalecer al SNI, donde el actor central es la empresa, las políticas de nanotecnología en Argentina aún se parecen más al modelo de Sábato y Botana, donde existen tres tipos de actores (Estado, comunidad científico tecnológica y empresas). En su modelo, el Estado ocupa el rol central, tal como ocurre con la nanotecnología en el caso argentino. Esto puede deberse a que el organismo principal de promoción de la nanotecnología ha sido un ministerio orientado a la comunidad científica y tecnológica, y no un ministerio de industria. A su vez, al hecho de que la inversión en I+D en Argentina es mayoritariamente realizada por el Estado (72\%), mientras que las empresas sólo aportan un $24 \%$. Éstos son porcentajes opuestos a aquéllos que se encuentran en los países de donde emana el modelo del SNI. Por ejemplo, en EUA el gobierno financia el 31\% de la inversión en I+D, mientras que las empresas, explican, un $62 \% .{ }^{7}$ El esquema de la figura 1 resalta estas conexiones y señala las influencias en la creación de dicho modelo. Específicamente, se destaca el accionar de los tres actores principales. En primer lugar, las instituciones de políticas públicas en CTI que han definido las nanotecnologías

7 Los datos corresponden al año 2011 y fueron obtenidos de <http://www.ricyt.org/>. 
FIGURA 1.

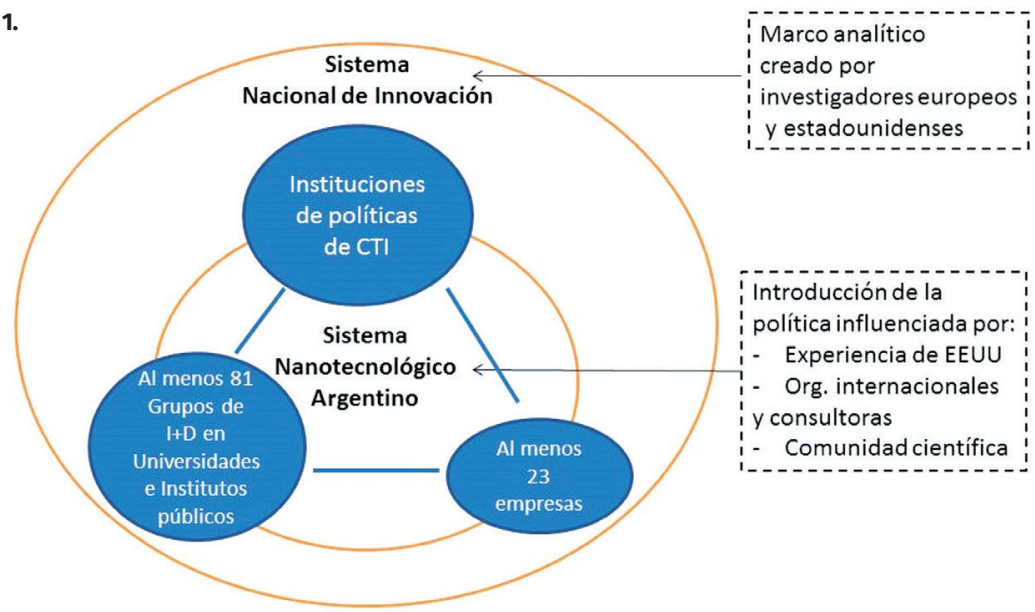

Fuente: Adaptación de Vila Seoane (2011).

como una prioridad y la apoyan financieramente. Esto incluye a: MINCyT; AGENCIA; CONICET; FAN. En segundo lugar, a los diversos institutos o universidades donde trabajan grupos de $\mathrm{I}+\mathrm{D}$ de nanotecnología. Por último a las empresas de origen de capitales argentinos que cooperan con grupos de I+D nacionales para el desenvolvimiento de nanotecnologías.

De la introducción del marco analítico del SNI, se desprenden algunas características del proyecto político de la nanotecnología en Argentina. Su punto fuerte por el momento ha sido la financiación de grupos de $\mathrm{I}+\mathrm{D}$, pero el mayor interés está en incrementar las conexiones con el sector empresario a fin de actualizar y fortalecer las capacidades industriales nacionales. Esta asociación de la política de ciencia y tecnología con políticas industriales no es casualidad, y acompaña a las principales tendencias de políticas industriales de Argentina que han definido un plan estratégico para el año $2020^{\circ}$ priorizando determinados sectores. Para varios miembros de la comunidad científica ésta es una buena noticia, pues busca conectar las actividades de investigación con las muchas veces vilipendiada industria nacional, pero no para todos. Sin duda también implica una sujeción de las prioridades de investigación a las necesidades industriales del país, ya que con base en criterios de "utilidad práctica" se amenaza la existencia de líneas de investigación que, aparentemente o por el momento, no tienen una conexión directa con el mundo industrial. De todas maneras, aún las colaboraciones han sido más en el área científica que en la empresarial. Esto en parte por la relativa facilidad de cooperar entre grupos de investigación que con empresas en el desarrollo de nuevos conocimientos.

$8<$ http://www.conicet.gov.ar/>.

$9<$ http://www.industria.gob.ar/plan-estrategico-2020/>. 
La respuesta a la pregunta de por qué el marco analítico del SNI se focaliza en empresas, soslayando otro tipo de actores, la brinda el contexto de origen de dicho modelo. Se basa en la experiencia de EUA y de otros países "desarrollados", e intenta capturar cuáles fueron los principales factores que intervinieron en el crecimiento económico de dichos países. Pero en su intento, el modelo se abstrae de varias variables que sin duda han tenido influencia en las trayectorias de dichas naciones, como cuestiones geopolíticas y de construcción de capital bélico, por llamarlo de algún modo, que se entrelazan con el crecimiento económico y el desarrollo científico tecnológico. Al trasladar sus recomendaciones a países latinoamericanos, en seguida surgen discrepancias por ser ideas fuera de contexto, marcos conceptuales diseñados para otras realidades, y absorbidos acríticamente (Thomas y Dagnino, 2005), en zonas que nunca satisfacen todas las condiciones necesarias para funcionar como en sus lugares de origen. Sin duda, no es lo mismo comparar Alemania, Israel, Estados Unidos o Corea del Sur, con cualquier país de Latinoamérica o de África subsahariana. Las diferencias no son sólo económicas, sino de trayectorias históricas, políticas, culturales, sociales, entre muchas otras, que precisan de marcos analíticos adecuados a las condiciones locales. Esto ya está en discusión, por ejemplo, con las iniciativas que hablan de la necesidad de apoyar "tecnologías sociales" (Dagnino, 2004). Sin embargo, y a pesar de su importancia por ser conceptos más arraigados en el territorio, han tenido poca influencia en las principales políticas de CTI, entre las cuales se incluye la nanotecnología. A partir de esta reflexión, es fácil entender por qué hay un tipo de actor sistemáticamente ausente en estos últimos años en las políticas de CTI: las organizaciones sin fines de lucro. Es entendible que no todas las posibles áreas de desarrollo de la nanotecnología se relacionen con ellas. Pero es innegable que hay varias con un gran potencial para atacar problemas de inclusión social y ambiental. Pero, al no incluirlas en los espacios y procesos de decisión de prioridades, se llega a un vacío y a una pobreza de visiones y perspectivas sobre el futuro que las tecnologías podrían seguir. La nanotecnología es una de las tantas áreas de CTI, donde este tipo de actores se encuentran ausentes.

\section{Tensiones en el nanomundo}

La introducción de una política para la nanotecnología en Argentina provocó una serie de tensiones. Entre ellas: la disputa por su definición; las dificultades en los vínculos entre empresas y grupos de $\mathrm{I}+\mathrm{D}$, y, los límites relacionados con la institucionalidad a nivel nacional.

\section{La disputa por su definición}

Al conversar con científicos sobre la definición de la nanotecnología, parecería existir cierta convergencia en que se trata del campo que estudia las 
nuevas propiedades de la materia originadas a escala nanométrica (Vila Seoane, 2011: 2). Aunque cuando se analizan con más detalle los términos de referencia de distintas convocatorias, tanto de una orientación más bien básica como de desarrollo de aplicaciones con el sector privado, es fácil encontrar definiciones ambiguas o que directamente se omitan. Por ejemplo, si bien la convocatoria de los fondos sectoriales habla de "propiedades de la materia a nanoescala", no se especifica que se trata de las "nuevas" propiedades que surgen en ciertos rangos de esa escala. ${ }^{10}$ Otro caso es la convocatoria de aportes no rembolsables del FONTAR Bio-Nano-TIC, donde se aclara que son elegibles proyectos de "Nanotecnología: nanomateriales, nanointermediarios y nanosensores", pero en ningún momento define qué se entiende por esos términos, a pesar de ser la nanotecnología una de las prioridades del instrumento. ${ }^{11}$

¿Cómo se puede entender esto? Una posible respuesta la brinda el análisis de políticas públicas. Siguiendo a Stone, el corazón de las mismas se centra en la discusión por la definición del concepto de un dado tema a apoyar (Stone, 1988: 7), ya que esto, de cierta forma, determina a quiénes se incluirá como beneficiarios de la política, y una definición imprecisa permite abarcar más destinatarios. En el caso de Argentina, organizaciones como la FAN suelen usar el término "micro y nanotecnologías". Y esto no es casualidad, pues se busca darle importancia a la comunidad en microtecnologías en el país, que cuenta con un desarrollo considerable en diversos centros de investigación, como por ejemplo la $\mathrm{CNEA}^{12}$ o el INTI. ${ }^{13}$ Es posible esperar similares disputas por el término en otros países, que dependerán de la estructura de la red de actores con más influencia. El caso argentino ilustra esto. Dado que la nanotecnología se estableció como un campo de investigación prioritario a nivel nacional, se destinaron recursos al área que atrajeron el interés de diversos grupos de investigación, muchos de los cuales no se encontraban previamente trabajando en temáticas a esa escala. La disponibilidad de recursos los llevó a reorientar sus líneas de I+D para presentarse a las distintas convocatorias. Estas tendencias sugieren que la nanotecnología, como término, se convirtió en una especia de concepto de frontera (Mollinga, 2010), es decir, en uno de aquellos conceptos polisémicos que pueden, a partir de su indefinición, incluir diverso tipo de actividades, a veces contrarias a su definición original.

10 Ver el anexo I de la convocatoria en: <http://www.agencia.mincyt.gob.ar/upload/Bases_ FSNano_2010.pdf >.

11 Ver la página 2 de la convocatoria en: <http://www.agencia.mincyt.gob.ar/upload/_Bases_ANR_BIO-NANO-TICs_2012_CII.pdf $\rangle$.

$12<$ http://www.cnea.gov.ar/>.

$13<$ http://www.inti.gob.ar/>. 


\section{Dos mundos aparte: las empresas y los "grupos" de I+D}

Los resultados de la encuesta ${ }^{14}$ realizada por el MINCyT reflejan los desafíos que enfrentan los grupos de I+D y las empresas argentinas de nanotecnología al trabajar en conjunto. Uno de sus resultados muestra tanto convergencias como divergencias en el tipo de aplicaciones industriales de las empresas o de las posibles líneas de I+D de los grupos. Por un lado, tanto grupos como empresas coincidieron en destacar la potencialidad de las redes nacionales de desarrollar productos en las áreas de salud humana, biotecnología y ambiente. Por otro lado, las empresas se inclinaron más por sectores que se ajustan a la realidad del perfil productivo del país, tales como autopartes, metalmecánica, agroindustria y alimentos, mientras que las respuestas de algunos grupos para esos mismos sectores fue bastante poco prioritaria (Vila Seoane y Rodríguez, 2012). Esto sugiere justamente la dificultad de varios grupos de $\mathrm{I}+\mathrm{D}$ de asociar sus líneas de investigación con las problemáticas industriales locales, y de cómo sus conocimientos podrían fortalecer estos sectores industriales más tradicionales. Hecho que no es de extrañar, pues los resultados de la encuesta a grupos de I+D reflejan que sólo un $10 \%$ de las vinculaciones son con empresas, mientras que la gran mayoría (36\%) se realiza con universidades extranjeras (Ibid. 2012: 22). Lo cual también evidencia las dificultades en aplicar el marco analítico del SNI.

Más allá de esto, los grupos de I+D expresaron que, a pesar de sus conocimientos en el área, reciben pocas demandas desde las empresas para resolver sus problemas tecnológicos. Y cuando las reciben, que las empresas suelen tener dificultades para plantear tecnológicamente dichos problemas. Esto dificulta la creación de un lenguaje común para abordar los desafíos. También resaltan que las empresas en general tienen poco interés en participar en proyectos de I+D, tanto por el tiempo que pueden llegar a consumir como por la incertidumbre de los resultados a alcanzar. Las empresas entrevistadas reconocen estas deficiencias, y lo indispensable de incrementar las articulaciones con el sistema científico y tecnológico. Aunque no dejan de manifestar su posición crítica con respecto a estas vinculaciones, tal como se evidencia en la siguiente cita:

El gran desafío es que la mayoría de los científicos en Argentina empiecen a orientar sus esfuerzos al desarrollo de tecnología, generando prototipos de productos o materiales útiles para aplicaciones concretas y no, como hasta ahora, que la gran mayoría centra su carrera profesional en las publicaciones en revistas con referato de alto impacto. Este tipo de científicos o tecnólogos deberían tener la capacidad de transformar las investigaciones en aplicaciones concretas y no, como en muchos casos, en la publicación de patentes de cuestionable utilidad. (Ibid. 2012: 29)

14 Cabe remarcar que estuvo destinada a grupos de I+D y a empresas principalmente nacionales operando en el país, pero no consideró aquellas multinacionales que utilizan dichas tecnologías. 


\section{Las dificultades en apoyarla institucionalmente}

La implementación del proyecto de la nanotecnología en Argentina no estuvo exenta de desafíos. En la tabla 1 sintetizo los más relevantes que podrían inspirar formas de intervención en otros países de la región, con el recaudo de que se refieren a la implementación de la política tomando como base el marco analítico del SNI.

Los recursos financieros son siempre una de las principales limitantes. Si bien hubo un aumento con el paso del tiempo (Vila Seoane, 2011: 102), suele ser una demanda tanto de grupos de I+D como de empresas. En especial, los grupos reclaman mayor financiamiento para desarrollos tecnológicos. $\mathrm{Y}$ en esto coinciden con los empresarios, que consideran como un cuello de botella la escasez de recursos para proyectos que planean escalar prototipos. Esto es infrecuente, y, aún más, en áreas tecnológicas "novedosas". Los empresarios también destacaron que el país carece de capital de riesgo, uno de los elementos centrales en países donde "funciona" el SNI, como EUA o Israel.

Varias áreas de la nanotecnología, aunque no necesariamente todas, dependen del uso de distintos tipos de equipos. Durante el trabajo de campo realizado, fue evidente esta necesidad y la de contar con la infraestructura apropiada. Por ejemplo, con centros nacionales donde los equipos puedan ser compartidos por distintos grupos y empresas, pues esta forma de organización abarataría los costos. Otro requisito fue contar con facilidades para obtener repuestos de los equipos en caso de desperfectos, y de personal capacitado adecuadamente para su operación.

La disponibilidad de recursos humanos es otro desafío. Si bien la cantidad de investigadores se duplicó en los últimos años (Vila Seoane y Rodríguez, 2012: 35), aún es una comunidad pequeña para la cantidad de potenciales áreas de aplicación que muchos avecinan. La interdisciplinariedad como elemento en la formación es otra carencia, dado que los desarrollos requieren de especialidades que no siempre se encuentran en el área de

TABLA 1. Principales desafíos detectados durante la implementación del proyecto de nanotecnología en Argentina.

\begin{tabular}{|c|c|c|}
\hline \multicolumn{3}{|l|}{ PRINCIPALES DESAFÍOS } \\
\hline Recursos financieros & $\begin{array}{l}\text { Articulación público- } \\
\text { privada }\end{array}$ & $\begin{array}{l}\text { Legislación (códigos aduaneros; } \\
\text { laborales; salud). }\end{array}$ \\
\hline Infraestructura y equipos & Desarrollo de mercados & $\begin{array}{l}\text { Difusión y sistema de } \\
\text { información }\end{array}$ \\
\hline $\begin{array}{l}\text { Recursos humanos e } \\
\text { interdisciplinariedad }\end{array}$ & $\begin{array}{l}\text { Cooperación } \\
\text { internacional }\end{array}$ & Ética y percepción pública \\
\hline
\end{tabular}

Fuente: Con base en Vila Seoane y Rodríguez (2012) y Vila Seoane (2011). 
experiencia de las disciplinas tradicionales en las que se organizan las universidades: química, física, medicina, electrónica, etc. Por ende, se han iniciado programas de movilización y capacitación de recursos en red, aunque de manera muy incipiente, y es otro de los temas a profundizar.

Si bien, como describí al comienzo, las políticas están siendo reorientadas para fomentar la articulación de actores del sistema científico y tecnológico con empresas, todavía es un vínculo escaso a nivel nacional. Esto sigue siendo un desafío por las diferencias culturales y de trabajo mencionadas en la sección previa. Un desafío que tendrá que sobrepasarse, por ejemplo, al compartir experiencias, buenas prácticas y metodologías de trabajo, al evaluar y corregir los proyectos que ya se han puesto en práctica.

Otro desafío es el poco desarrollo de mercados, tanto nacionales como internacionales. Los empresarios se lamentan de la escasa demanda de los consumidores de productos que abiertamente indiquen que utilizan nanotecnología. Una alternativa que no se ha experimentado aún, es la implementación de regulaciones por parte del Estado que promuevan aplicaciones nanotecnológicas. Por ejemplo, esto podría fomentarse al requerir determinado tipo de prueba con nanosensores en alimentos, que generaría un mercado de proveedores y productores locales, que luego, incluso, podrían exportar sus desarrollos.

La difusión surgió como otro eje desafiante para la promoción del área. Un término poco común para el público en general, y para muchos empresarios tampoco es clara su posible aplicación en su proceso industrial. Por ende, los esfuerzos de difusión en diversas esferas es uno de los puntos esenciales para un crecimiento de las actividades relacionadas con la nanotecnología. Lo anterior, incluye tanto la difusión de las posibles aplicaciones que las líneas de investigación en curso puedan llegar a tener, así como también la comunicación de casos exitosos de empresas que podrían incentivar a otras a entrar en esta área. También se señaló como una falencia la falta de un sistema de información actualizado en ésta y en otras áreas estratégicas. Algo que los países líderes en la temática tienen, como Alemania ${ }^{15}$ o el Reino Unido. ${ }^{16}$

Argentina ha realizado avances en términos de cooperación internacional al establecer centros binacionales con Brasil, ${ }^{17}$ México ${ }^{18}$ y Sudáfrica, ${ }^{19}$ que permiten estrechar vínculos binacionales y promover la transferencia de conocimientos. Sin embargo, aún es un área que se puede expandir de forma

$15<$ http://www.nano-map.de/>.

$16<$ http://www.nano.org.uk/>.

17 <http://www.mincyt.gob.ar/accion/cabnn-centro-argentino-brasileno-de-nanociencias-ynanotecnologia-6453>.

18 <http://www.mincyt.gob.ar/accion/camen-centro-argentino-mexicano-de-nanociencia-ynanotecnologia-9565>.

19 <http://www.mincyt.gob.ar/accion/asacen-centro-argentino-sudafricano-de-nanotecnologia-9564>. 
más estratégica, por caso, al promover vinculaciones que generen capacidades nacionales al absorber conocimientos específicos para la resolución de problemas.

Otros puntos vacantes se relacionan con la ausencia de legislación en diversas áreas. Esto incluye desde la actualización de los códigos aduaneros para catalogar adecuadamente la exportación e importación de productos nanotecnológicos, como la creación de regulaciones destinada a lugares de trabajo donde se utilicen nanomateriales para limitar potenciales efectos nocivos para la salud. Esto no sólo tendría que considerar investigaciones y productos locales, sino también aquellos que podrían ser importados. Pues, al no estar regulados, tienen el potencial de ser usados de manera indiscriminada en el país soslayando su efectos tóxicos.

Finalmente, el tema de la ética en investigaciones de nanotecnología y su percepción pública sigue siendo un área escasamente explorada. Y casi siempre con menor prioridad. No obstante, un descuido en el área puede producir efectos boomerang que bloqueen desarrollos futuros, tal como ocurrió con la biotecnología.

\section{Discusión}

La nanotecnología como política pública se desarrolló en Argentina tanto por factores internos como externos. Entre los externos se destacó el impulso a nivel mundial causado por la iniciativa de EUA, los organismos internacionales de crédito, consultoras y la comunidad científica internacional, que desparramaron la idea por el mundo al posicionar a la nanotecnología como la futura área del conocimiento que revolucionará la producción industrial. Estrategia similar a la que se usó en su momento con las biotecnologías y las TICs, o la ola de promesas que acontecen en estos días con el Big Data. Con la diferencia de que los aportes prácticos de la nanotecnología son menos visibles para los consumidores. No existe un caso tan visible como el de la PCs para las TICs o de la oveja Dolly para biotecnología. En cuanto a los factores internos, se destacó la influencia de la comunidad científica nacional, conectada con redes globales, e influenciada por las discusiones y las promesas existentes en otras latitudes. Este hecho marcó la forma de promoción inicial por parte del Estado. Financiamiento dirigido principalmente a grupos de $\mathrm{I}+\mathrm{D}$, pero tendiendo progresivamente en el tiempo a estimular vínculos con el sector privado. Estas alianzas no estuvieron ausentes de desencuentros, dadas las diferentes prácticas de los grupos de $\mathrm{I}+\mathrm{D}$ y las empresas. Otro punto a destacar de la introducción de la nanotecnología, como una prioridad a nivel ministerial en Argentina, es que terminó usando un concepto difuso, que pocas veces se relaciona con su definición científica original en las convocatorias. En cambio, la nanotecnología se convirtió en una palabra polisémica con la posibilidad de incluir tecnologías diversas y distantes entre sí; como, por ejemplo, la microtecnología. Hecho que refleja la disputa por 
los recursos financieros disponibles por los actores dentro de la comunidad científica y tecnológica.

El caso argentino evidencia que dentro de las limitaciones existentes en países de ingresos medios es posible construir capacidades en una nueva área emergente como la nanotecnología. Es indudable que en 10 años se pasó de no tener casi nada dedicado al área, a crear un conjunto incipiente de actores, más o menos conectados, con diversas capacidades de generación y absorción de conocimientos. Por lo tanto, y partiendo desde el marco de una política orientada a fortalecer al "sistema nacional de innovación", se puede decir que hubo avances, aunque lentos, en términos de pasar de apoyos sólo de grupos de I+D, a alianzas público-privadas. Los resultados muestran que se cuenta con una comunidad de al menos 629 investigadores en el tema, y una de 23 empresas asociadas con los instrumentos estatales (Ibid., 2012). Es coherente suponer que el número total de aquellas trabajando con nanotecnologías es mayor. Esto es un resultado de las decisiones de intervención gubernamental de los últimos años, que si bien lejanas del ideal de muchos, son mejor que nada y han permitido construir capacidades mínimas en ambas áreas. Queda como desafío incrementar la intensidad de las relaciones, y la capacidad de que verdaderamente se introduzcan productos en las distintas cadenas de valor industriales. No obstante, son evidentes las tensiones y limitaciones que se tiene al adoptar un marco analítico, como el SNI, para las políticas surgidas en otro contexto, y que no se ajustan del todo bien a las realidades socioeconómicas de Argentina. Existen condiciones de borde que imponen restricciones estructurales en términos de recursos (tanto financieros, humanos como de infraestructura), e institucionales que no se pueden cambiar en un santiamén, y sintetizados en los 11 puntos mencionados en la cuarta sección: recursos financieros; infraestructura y equipos; recursos humanos y estímulo a la interdisciplinariedad; articulación público-privada; el desarrollo de mercados; la cooperación internacional; introducción de nuevas legislaciones; difusión de las potencialidades de las nanotecnologías y generación de sistemas de información; sin dejar de lado las investigaciones sobre la ética en investigaciones de nanotecnología y sobre su percepción pública.

Como se vislumbra en la figura 1, el marco analítico del SNI se parece más al triángulo de Sábato y Botana, donde el Estado tomó el rol central en la relación de los tres tipos de actores considerados. El sistema es una metáfora para las conexiones, los proyectos, los actores, entre otros, que han surgido en los últimos años en torno a la nanotecnología. Esta configuración delinea claramente la orientación para la cual la gran cantidad de recursos (humanos y materiales) está siendo utilizada en Argentina, para contribuir con los procesos industriales, y así aportar al "desarrollo económico" de la Nación. Pero en países donde las conexiones con el sector empresarial son escasas, donde las necesidades de innovaciones volcadas a la resolución de asuntos sociales son indispensables, el foco exclusivo en el sector privado 
pone en duda el esquema mental de organización de la política y de su flujo de fondos, pues se deja de lado todo el otro conjunto de posibles aplicaciones de la nanotecnología que tantas veces figuran en la literatura para aliviar las condiciones de sectores con menos recursos y contribuir a resolver los problemas ambientales. Temas difícilmente incluidos en la agenda si no se abren espacios para la participación activa de representantes de la sociedad civil. Una de las posibles causas de esto, más allá de las limitaciones de los marcos analíticos utilizados, puede tener que ver con el pequeño tamaño de la comunidad que trabaja con nanotecnología a nivel nacional, ya que esto es un límite para la cantidad de proyectos o áreas en las cuáles los grupos de I+D pueden trabajar. Y, hoy por hoy, los incentivos están orientados a estimular conexiones con el sector privado. ¿Cómo corregir esta deficiencia? La participación de otros actores en la definición de las políticas públicas sería el primer paso. Esto tendría que estar acompañado por instrumentos de financiamiento que también guíen las investigaciones para la realización de proyectos en conjunto con tales organizaciones, y no sólo con empresas privadas. De lo contrario, el proyecto de la nanotecnología en Argentina tiene algunas posibilidades de aportar al desarrollo económico de las empresas nacionales, pero muy pocas de contribuir con procesos de inclusión social o de cuidado del ambiente por intermedio de la acción de la rica variedad de organizaciones sin fines de lucro existentes en el país, como cooperativas, movimientos sociales u otras organizaciones sin fines de lucro.

Para concluir, quiero resaltar que las recientes investigaciones consideran la innovación como un proceso social, que depende de los conocimientos acumulados por todas las generaciones previas (Alperovitz y Daly, 2008). En el caso de los países lejanos a los principales centros de producción de conocimiento, la capacidad de innovar depende, además, de qué tan buenos sean en absorber nuevas prácticas, y en adaptarlas a la realidad local. Es por ello que si bien es frecuente encontrar artículos que vinculan "nanotecnología" y sus posibles aplicaciones en "países en desarrollo", será difícil que algo de esto se vuelque de forma sistemática a la práctica sin un cuestionamiento y una profunda reformulación en los marcos analíticos que guían el diseño de políticas, y que reorienten la forma en la que el conocimiento se produce y se utiliza. Para ello, el papel de la comunidad científica abocada a las nanotecnologías tiene una función importante que cumplir. No sólo para continuar desarrollando investigaciones en la temática, sino también para ser conscientes de que para lidiar con los problemas sociales y ambientales de la región, se necesita extender la cantidad de actores presentes en la formulación de las políticas de CTI, y en expandir las formas de difusión de conocimientos en la "sociedad". Latinoamérica, en los últimos 10 años ha experimentado varios cambios y desafíos a la recepción de modelos externos, y realizado nuevas propuestas como el Buen Vivir (Gudynas, 2011). No obstante, estas discusiones todavía no han repercutido con toda su fuerza en las nuevas áreas de investigación. Tema pendiente a fin de llegar a diseñar 
sistemas de investigación y desarrollo más inclusivos y coherentes con las condiciones regionales.

\section{Bibliografia}

Alperovitz, G. y Daly, L. (2008). Unjust deserts: How the rich are taking our common inheritance. Nueva York: The New Press.

Andrini, L. y Figueroa, S. (2008) El impulso gubernamental a las nanociencias y nanotecnologías en Argentina. En G. Foladori y N. Invernizzi (eds.) Las nanotecnologías en América Latina. México DF: Miguel Ángel Porrúa.

Dagnino, R. (2004) A tecnologia social e seus desafios. En Tecnologia social - uma estratégia para o desenvolvimento. Río de Janeiro: Fundação Banco do Brasil.

Escobar, A. (1995) Encountering development. The making and unmaking of the Third World. Princeton, New Jersey: Princeton University Press.

Freeman, C. (1995) The 'National System of Innovation' in historical perspective. Cambridge Journal of Economics, (marzo, 1993): 5-24.

Godin, B. (2006) The linear model of innovation. The historical construction of an analytical framework. Science, Technology \& Human Values, 31(6): 639-667.

Gudynas, Eduardo (2011) Buen vivir: Today's tomorrow. Society for International Development, 54(4): 441-447.

Hornidge, A.-K. (2012) 'Knowledge' in development discourse: critical review. En A.-K. Hornidge y Ch. Antweiler (eds.) Environmental uncertainty and local knowledge Southeast Asia as a laboratory of global ecological change. Bielefeld: Transcript Global Studies.

Krause, U. (2013) Innovation: the new big push or the post-development alternative? Society for International Development, 56(2): 223-226.

Lundvall, B.-Å., Johnson, B., Andersen, E. S. y Dalum, B. (2002) National systems of production, innovation and competence building. Research Policy, 31(2): 213231.

MINCyT. (2009) BET - Nanotecnología. Buenos Aires, Argentina: Ministerio de Ciencia, Tecnología e Innovación. <http://www.mincyt.gob.ar/_post/descargar. php?idAdjuntoArchivo=22522 >.

Mollinga, P. P. (2010) Boundary work and the complexity of natural resources management. Crop Science, 50(1), 1-9.

Nelson, R. R. (ed.) (1993) National innovation systems: a comparative analysis. Oxford: Oxford University press.

Sábato, J. y Botana, N. (1970) La ciencia y la tecnología en el desarrollo de América Latina. En A. Herrera (ed.) América Latina: ciencia y tecnología en el desarrollo de la sociedad. Santiago de Chile: Editorial Universitaria.

Stone, D. (1988) Policy paradox and political reason. Glennview: Illinois: Scott Forseman.

Thomas, H. y Dagnino, R. (2005) Efectos de transducción : una nueva crítica a la transferencia acrítica de conceptos y modelos institucionales. Ciencia, Docencia y Tecnología, 31(XVI), 9-46. 
Mundo Nano | Artículos | vol. 7, No. 13, julio-diciembre, 2014 | www.mundonano.unam.mx

Vila Seoane, M. (2011) Nanotecnología: su desarrollo en Argentina, sus características y tendencias a nivel mundial, tesis de maestría. Universidad Nacional de General Sarmiento, Buenos Aires.

Vila Seoane, M. Y Rodríguez, S. (2012) Empresas y grupos de nanotecnología en Argentina. Buenos Aires, Argentina: Ministerio de Ciencia, Tecnología e Innovación Productiva. 